Article

\title{
IEC 61850-Based Centralized Busbar Differential Protection with Data Desynchronization Compensation
}

\author{
Myeong-Hoon Song ${ }^{1}$, Sang-Hee Kang ${ }^{1}{ }^{(0)}$, Nam-Ho Lee ${ }^{2}$ and Soon-Ryul Nam ${ }^{1, *}$ \\ 1 Department of Electrical Engineering, Myongji University, Yongin 17058, Korea; \\ mhs7234@gmail.com (M.-H.S.); shkang@mju.ac.kr (S.-H.K.) \\ 2 Korea Electric Power Research Institute, Daejeon 34056, Korea; namho.lee@kepco.co.kr \\ * Correspondence: ptsouth@mju.ac.kr; Tel.: +82-31-330-6361
}

Received: 5 January 2020; Accepted: 17 February 2020; Published: 21 February 2020

check for updates

\begin{abstract}
This paper proposes an IEC 61850-based centralized busbar differential protection scheme, in which data desynchronization between intelligent electronic devices (IEDs) leads to differential current errors. As the differential current errors could result in erroneous operation of the centralized busbar differential protection, data desynchronization should be compensated for. The main causes of data desynchronization are subdivided into measurement timing and time synchronization errors. In this paper, the first-order Lagrange interpolation polynomial is used to compensate for measurement timing errors and the voltage angle differences between IEDs are used to compensate for time synchronization errors. The centralized busbar differential protection is tested using a real-time digital simulator and IEC 61850-based IEDs, which are implemented with the MMS-EASE Lite library and Smart Grid Infrastructure Evaluation Module. The test results show that the data desynchronization compensation can significantly reduce differential current errors, and thus prevent erroneous operation of the IEC 61850-based centralized busbar differential protection.
\end{abstract}

Keywords: centralized busbar differential protection; IEC 61850; data desynchronization compensation; measurement timing error; time synchronization error

\section{Introduction}

Today, IEC 61850 is considered as the mainstream in communication networks for digital substations. The first version focused only on communication networks and systems in substations. However, the second edition was expanded to cover communication networks and systems for power utility [1]. The standard mandates integration of all protection, control, measurement, and monitoring functions of a power system network at both the process and station levels. In addition, several new features impact protection system design. For example, the authors of [2,3] used IEC 61850 to (respectively) adapt the $67 / 67 \mathrm{~N}$ protection of main ring systems featuring distributed energy resources and to improve the fault detection times of protection relays in different substations. IEC 61850 has also been usefully applied to microgrids and distribution networks [4-7]. The authors of $[4,5]$ proposed centralized protection strategies for microgrids and the authors of [6] proposed the design of microgrid plug-and-play functions in accordance with IEC 61850. In [7], the three-level coordinated control was proposed for voltage regulation in distribution networks and its effectiveness was validated by experiment through a real communication link. In South Korea, the Korean Electric Power Corporation (KEPCO) has already applied IEC 61850 to more than 52 digital substations [8].

IEC 61850-based centralized protection has been widely studied over the past several decades [9-19]. Synchronization of data received from intelligent electronic devices (IEDs) located in substations is 
essential. Although the methods of [9-11] enhanced protection system performance using centralized data have been described, synchronization was not addressed. The authors of [12,13] used a global positioning system (GPS) to maintain data synchronization. Although this worked well, additional investment was required. Moreover, error is possible if the GPS is jammed. The recent IEC/IEEE 61850-9-3 defined an IEEE Std 1588 Precision Time Protocol (PTP) to increase data synchronization precision [20]. The methods of [14-19] utilized this PTP to synchronize IED data precisely. However, the PTP is costly to implement and the possible jamming problem remains in its master clock.

Busbars on a substation transmission side are usually protected by differential protection but busbars on the distribution side are protected by only overcurrent protection because differential protection on that side is costly to implement and hardware complexity exists due to the large number of distribution feeders and their flexible configuration. Although the centralized busbar protections of $[18,19]$ deal with hardware complexity, the PTP mentioned above remains costly and subject to GPS jamming. In this paper, we use conventional time synchronization employing a simple network time protocol (SNTP); such synchronization can sometimes create an error of approximately 1 ms [21-23], in turn creating significant errors in the current differential. Even in the absence of such errors, data desynchronization may occur if there are measurement timing errors between IEDs. Here, in the first step of data desynchronization compensation, we use a Lagrange interpolation polynomial to compensate for measurement timing errors assuming that time synchronization is in fact perfect. The polynomial has been used to estimate missing data [24,25] and can compensate for measurement timing errors by approximating values lying between two data points. In the second step, time synchronization errors are compensated using the voltage angle differences between IEDs. IEC 61850 deals with Sampled Values (SV) and Generic Object-Oriented Substation Events (GOOSE) [26-28]. Typically, voltage and current data are transmitted from merged units on a process bus to IEDs on a station bus $[29,30]$. Here, we develop centralized, busbar differential protection using the IEDs on the station bus, where phasor data are transmitted as SVs. Network traffic is reduced when phasor data rather than instantaneously sampled data are transmitted. The centralized busbar differential protection with data desynchronization compensation is tested using IEC 61850-based IEDs and a Real-Time Digital Simulator (RTDS). The IEC 61850-based IEDs are implemented using the MMS-EASE Lite library (SISCO, Inc., Sterling Heights, MI, USA) and the Smart Grid Infrastructure Evaluation Module (SGIEVM; Texas Instruments, Inc., Dallas, TX, USA) [31,32].

The details of the data acquisition, proposed protection scheme, and test results will be discussed in the following sections. Section 2 will describe the IEC61850-based data acquisition, while centralized busbar differential protection with data desynchronization compensation will be covered in Section 3. Results and the conclusion will be provided in Sections 4 and 5, respectively.

\section{Data Acquisition for Centralized Backup Protection}

GOOSE and SV can be used as protocols for the acquisition of phasor data by a central IED, in which centralized protection is implemented as backup protection. It is difficult to periodically transmit phasor data using GOOSE, as these data are typically transmitted in response to an event; however, the retransmit function of GOOSE can improve the reliability of data transmission [33]. As GOOSE cannot be used to specify the transmission period, to transmit the phasor data periodically, they should be updated based on a fixed interval. For this, phasor data can be updated using a statistical calculation model defined in IEC 61850 (2nd Edition), which generates maximum, minimum, average, and total data during a specified period [34]. Compared with GOOSE, SV does not have a retransmission function, but the sampling period can be specified, and multiple data points can be transmitted in a single frame [35]. Although each protocol has advantages and disadvantages, phasor data exchange based on SV is used in this paper to improve compensation accuracy by transmitting multiple data points in a single frame. 


\subsection{Centralized Backup Protection}

Centralized backup protection will be slower than main protections. Therefore, frequent data exchanges are inefficient, and can have negative effects on the protection functions of existing IEDs under conditions of increased network traffic. In addition to the low rate of data exchange, it is beneficial for centralized backup protection to exchange voltage and current phasor data rather than instantaneously sampled data. Phasor measurements require that the signal is sampled over a certain period, and the phasor cannot be measured correctly when any of the instantaneously sampled data are lost. If each IED transmits its phasor data instead of the instantaneously sampled data, the total data volume will be reduced as the phasor data describes the entire period of the instantaneously sampled data, and the computational burden of the central IED will also be reduced. In addition, phasor data can be transmitted with a relatively long period, and with the centralized backup protection still maintained within the timescale of backup protection. Figure 1 shows the structure of the centralized backup protection. Each local IED transmits its phasor data to the central IED using SV, and the central IED provides backup protection based on the phasor data received.

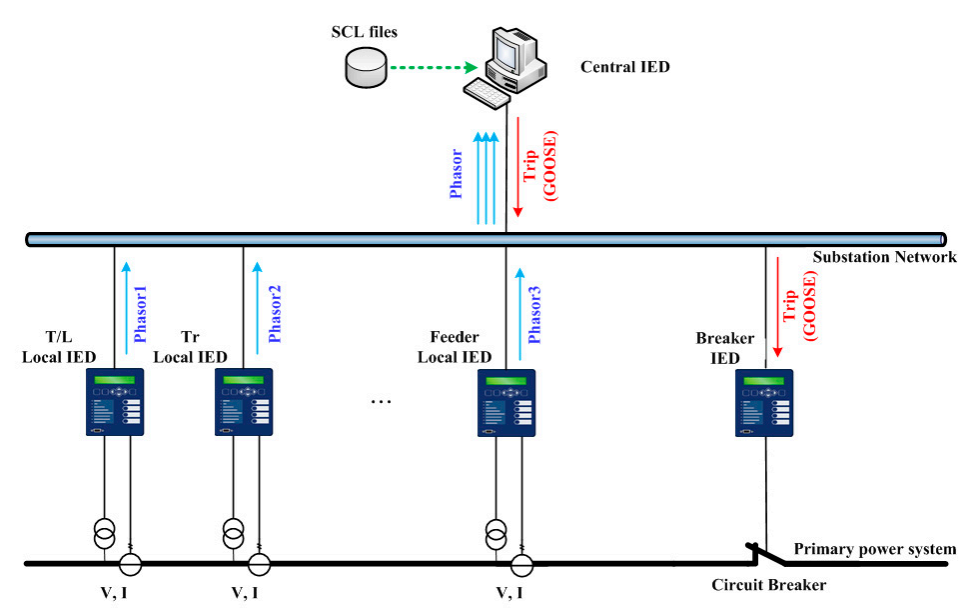

Figure 1. Centralized backup protection with phasor data exchange.

\subsection{Phasor Data Acquisition Using IEC 61850-9-2}

The SV protocol is typically used to exchange voltage and current data; its dataset includes not only the data of the common data classes (CDCs) [36] in the voltage and current transformers (TCTR and TVTR, respectively) [37], but also the data of other CDCs. The IEC 61850-9-2 light edition (LE) defines an SV scheme that follows the guidelines published by the Utility Communication Architecture International Users Group (UCAIug) [38]. The SV protocol, in conformance with IEC 61850-9-2LE as summarized in Table 1 , is dictated by a pre-defined dataset on the merging unit connected to the voltage and current transformers (CTs), which produces instantaneously sampled data at a rate of 80 or 256 samples per cycle and transmits 8 samples per frame. For example, 80 samples per $60 \mathrm{~Hz}$ corresponds to a frame transmission interval of $1.67 \mathrm{~ms}$, according to the number of application service data units (ASDUs).

Table 1. Comparison of IEC 61850-9-2 and IEC 61850-9-2LE.

\begin{tabular}{ccc}
\hline Area & IEC 61850 9-2 & IEC 61850 9-2 LE \\
\hline Sampling rate & Free parameter & $\begin{array}{c}\text { 80 samples/period (protection and metering) } \\
\text { 256 samples/period (power quality) }\end{array}$ \\
\hline \multirow{2}{*}{ Dataset } & Configurable & $\begin{array}{c}\text { 3 phase current + neutral current } \\
\text { 3 phase voltage + neutral voltage }\end{array}$ \\
\hline Time synchronization & Not defined & Optical pulse per second (1PPS) \\
\hline
\end{tabular}


In this paper, the centralized backup protection uses the phasor data instead of the instantaneously sampled data defined in IEC 61850-9-2LE. Therefore, the SV protocol in conformance with IEC 61850-9-2 is selected, as phasor data can be exchanged, and the sampling rate can be configured.

Phasor data were transmitted every $50 \mathrm{~ms}$ by setting the sampling mode (SmpMod) to "SAMPLES_PER_SECOND," and the sampling rate (SmpRate) to 100. The number of units (nofASDU) was set to five to transmit phasor data measured at 10-ms intervals. As shown in Figure 2, the oldest data are stored in ASDU1, and the next most recent are stored in ASDU2, as determined by SmpRate. Typically, three-phase voltage and current readings are included in the dataset, but the dataset in this paper included only the A-phase data for the purpose of simplification.

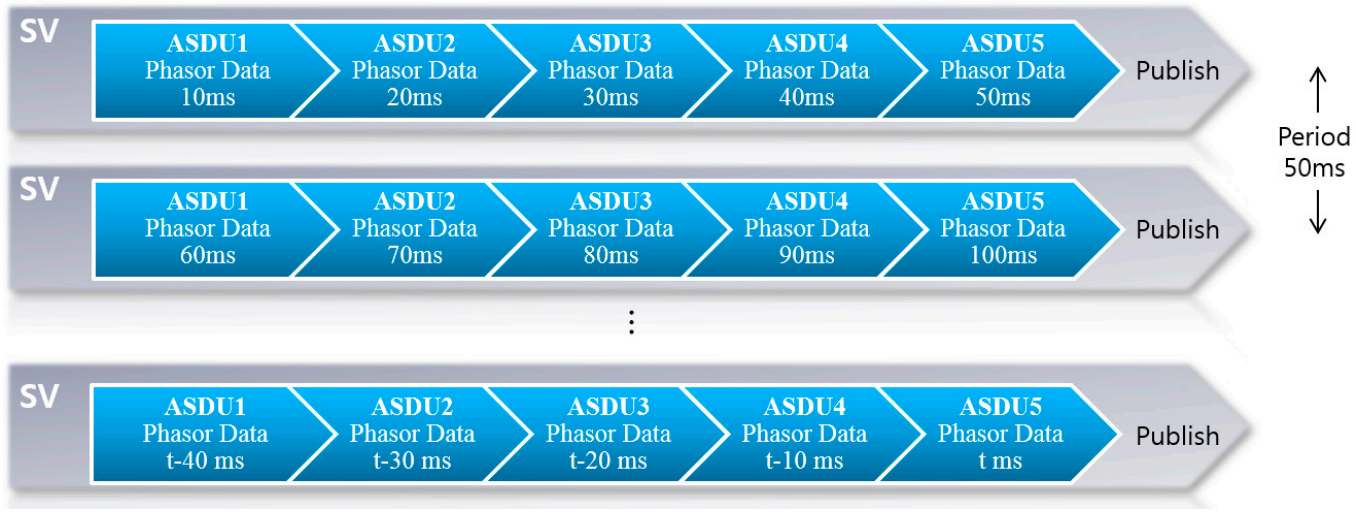

Figure 2. Phasor data of sampled values (SV) transmitted in conformance with IEC 61850-9-2.

\section{Centralized Busbar Differential Protection}

Differential protection utilizes the vector sum of current phasors with a minimum pickup offset to prevent improper operation. If the minimum pickup offset is increased considering data desynchronization, the detection sensitivity will be decreased. In the existing busbar protection scheme shown in Figure 3a, as analog current signals are measured simultaneously, current phasors are naturally synchronized. However, for the phasor-driven centralized protection scheme shown in Figure $3 b$, data synchronization should be carefully considered. In this case, local IEDs transmit phasor data through a network, so differential current errors can occur if their phasor data are not synchronized.

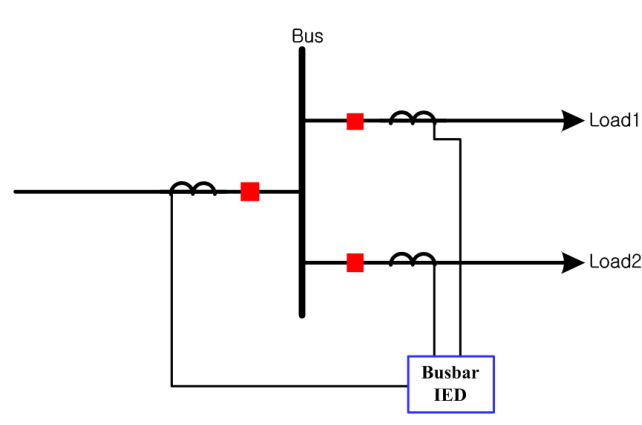

(a)

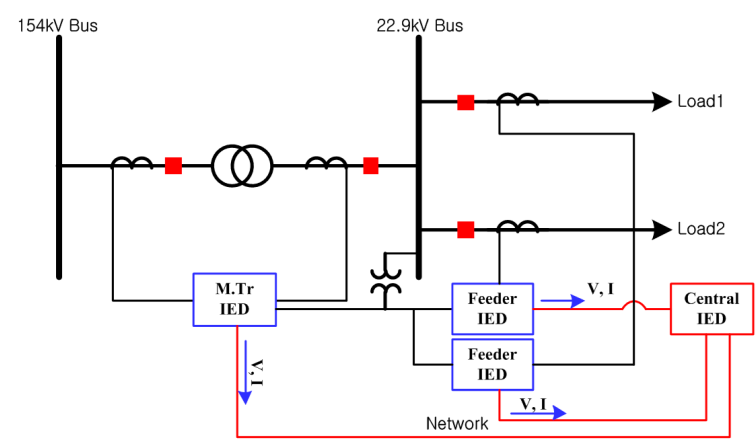

(b)

Figure 3. Comparison of the (a) existing and (b) centralized busbar differential protection schemes.

The phasor data measured at each IED may represent different timeframes, which can cause the central IED to operate erroneously. Usually, millisecond-scale errors can occur in SNTP; an error of $1 \mathrm{~ms}$ results in a $21.6^{\circ}$ phase shift in a $60 \mathrm{~Hz}$ system, which would be sufficient to activate current differential protection. 


\subsection{Data Desynchronization Compensation}

The main causes of data desynchronization can be classified as measurement timing and time synchronization errors. Measurement timing errors occur when data sources update at different times, and time synchronization errors occur when there is an error in the associated timestamp. Even when there is an external fault or no fault, differential current protection can be triggered due to data desynchronization, which should be compensated for.

If local IEDs are updated simultaneously, it is not necessary to compensate for measurement timing errors. Unfortunately, the measurement time is dependent on local timers or an external trigger signal. In this paper, the first-order Lagrange interpolation polynomial is used to compensate for measurement timing errors. After compensation of the measurement timing errors, time synchronization errors can be calculated by comparing the voltage angles received from each local IED, which should be equal if there is no time synchronization error.

\subsubsection{Compensation for Measurement Timing Errors}

In this paper, the first-order Lagrange interpolation polynomial was used to compensate for the measurement timing errors; this is because a higher-order Lagrange interpolation polynomial would cause some additional errors when there is a sudden change in the data measured. Generally, the Lagrange interpolation polynomial is defined as:

$$
f_{n}(x)=\sum_{i=0}^{n}\left(L_{i}(x) \times f\left(x_{i}\right)\right) \quad \text { with } L_{i}(x)=\prod_{i=0, j \neq i}^{n} \frac{x-x_{j}}{x_{i}-x_{j}}
$$

where $x$ is the time axis and $x_{i}$ is the $i^{\text {th }}$ datum. Therefore, the first-order Lagrange interpolation polynomial is given by:

$$
f_{1}(x)=\frac{x-x_{1}}{x_{0}-x_{1}} \times f\left(x_{0}\right)+\frac{x-x_{0}}{x_{1}-x_{0}} \times f\left(x_{1}\right)
$$

Figure 4 illustrates the compensation for the measurement timing errors using interpolation.

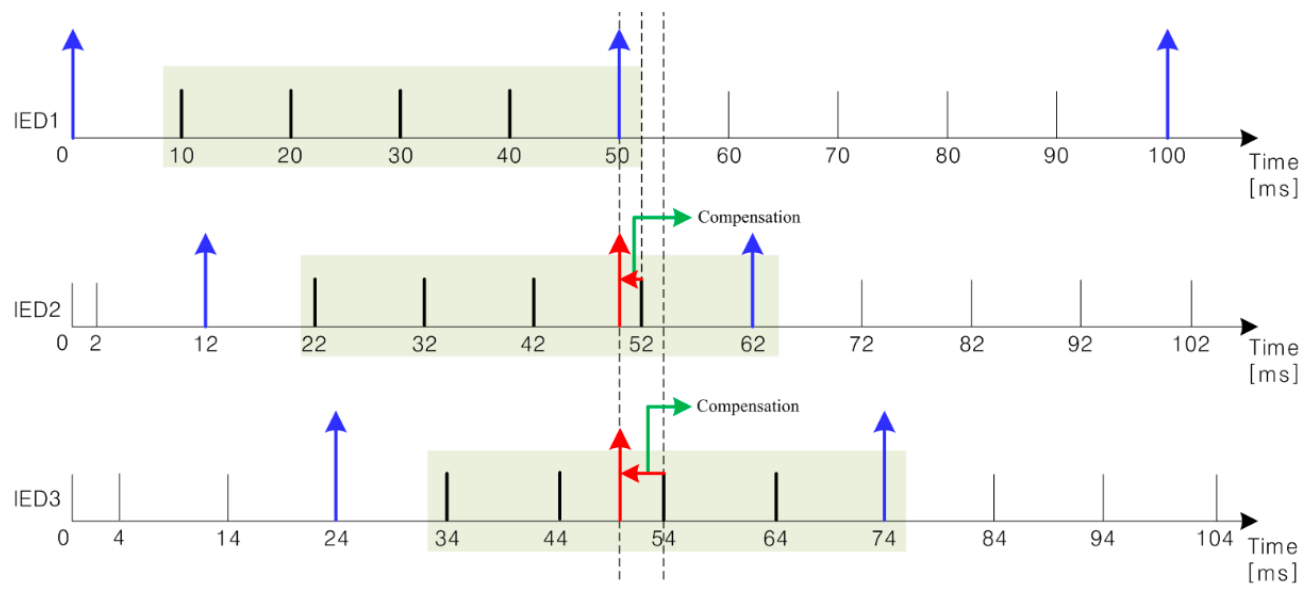

Figure 4. Compensation for the measurement timing errors among local intelligent electronic devices (IEDs).

A phasor is composed of magnitude, angle, and timestamp. A first-order Lagrange interpolation can be easily used to determine the magnitude, but the angle is discontinuous as it wraps from $180^{\circ}$ to $-180^{\circ}$, which complicates the angle compensation. The angle between the $n-1^{\text {th }}$ and $n^{\text {th }}$ datum is linearized by adding an offset angle to the angle of the $n^{\text {th }}$ datum before the angle interpolation is implemented. Equations (3) and (4) describe how the offset angle was calculated. While the angle at the $n^{\text {th }}$ datum, $\theta_{n}$ is measured, it can be also calculated using the time interval and the angle at the $n-1^{\text {th }}$ datum. 


$$
\delta_{n}=\theta_{n-1}+\left(t_{n}-t_{n-1}\right) \times \frac{1}{60} \times 360^{\circ}
$$

where $\delta_{n}$ is the calculated angle at the $n^{\text {th }}$ datum and $\theta_{n-1}$ is the measured angle at the $n-1^{\text {th }}$ datum. Assuming that the timestamp is correct, the offset angle will be a multiple of $360^{\circ}$. Therefore, it is obtained in integer multiples of $360^{\circ}$ degrees:

$$
\text { offset }_{\mathrm{n}}=\operatorname{TRUNC}\left(\frac{\delta_{n}-\theta_{n}}{360^{\circ}}\right) \times 360^{\circ}
$$

where the TRUNC function removes the fractional part of a number and truncates a number to an integer. Figure 5 illustrates that the offset angles are in multiples of $360^{\circ}$ at the angle discontinuities. Angle interpolation is performed after this offset angle is added to $\theta_{n}$.

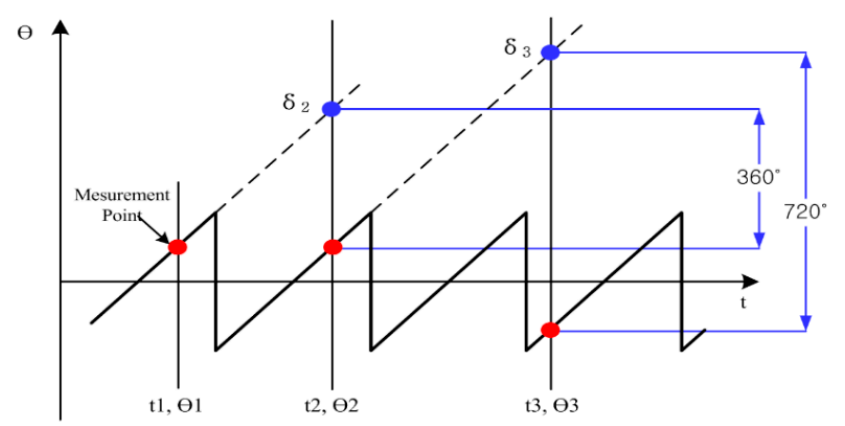

Figure 5. Angle discontinuity with offset angles in multiples of $360^{\circ}$.

\subsubsection{Compensation for Time Synchronization Errors}

Figure 6 shows that the voltage angles of three local IEDs have time synchronization errors after the measurement timing errors have been compensated for.

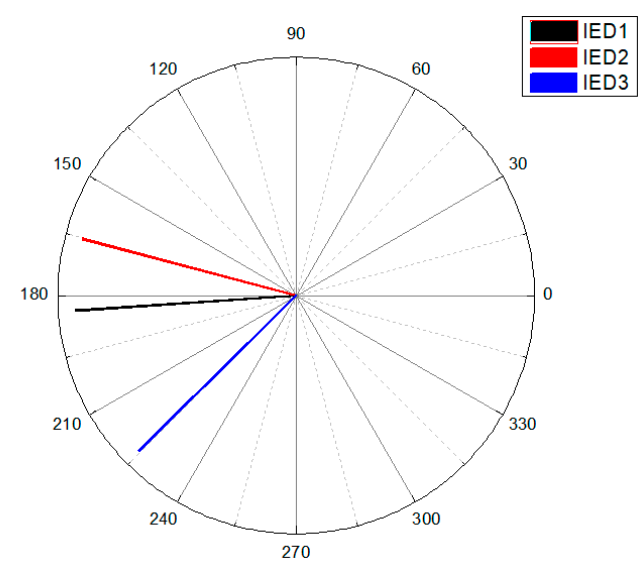

Figure 6. Detection of time synchronization errors using voltage angles.

As the potential transformer supplies the same voltage signal to each local IED, their voltage angles should be identical. However, time synchronization errors lead to voltage angle differences, which are used to compensate for time synchronization errors. Thus, a reference IED is defined as the local IED that has the voltage angle with the greatest lag, which is selected by:

$$
\text { Index } M \text { of } \operatorname{IED}_{r e f}=\operatorname{Max}\left(\theta_{1}^{v}, \cdots, \theta_{n}^{v}, \cdots, \theta_{N}^{v}\right)
$$

where $\theta_{n}^{v}=\angle\left(V_{1} \times V_{n}^{*}\right)$ indicates the voltage angle difference between the first and $n^{\text {th }}$ IED. After selecting the reference IED, any time synchronization error can be calculated based on the voltage 
angle difference between the reference IED and a leading IED. The time synchronization error of each IED is compensated for by interpolating its current phasor.

In a $60 \mathrm{~Hz}$ system, the time point used for interpolation is calculated by:

$$
t_{\text {intp- } n}=t_{n}+\frac{1}{60} \frac{\angle\left(V_{M} \times V_{n}^{*}\right)}{360^{\circ}}
$$

Figure 7 illustrates how to calculate the time synchronization errors.

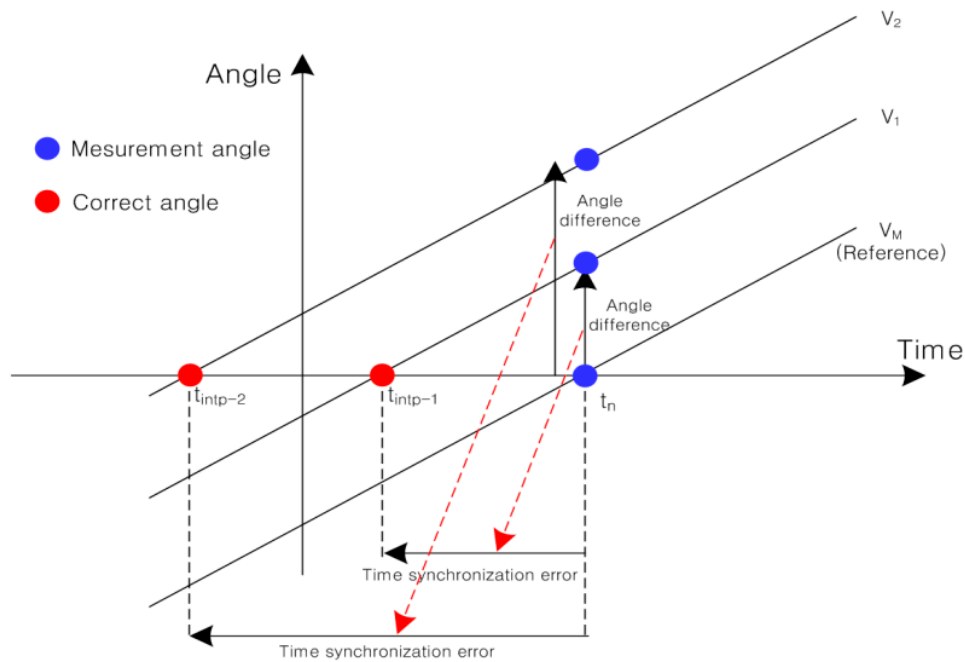

Figure 7. Compensation of time synchronization errors using busbar voltage angles.

\subsection{Centralized Busbar Differential Protection with Desynchronization Compensation}

Figure 8 shows a flowchart of the centralized busbar differential protection proposed herein.

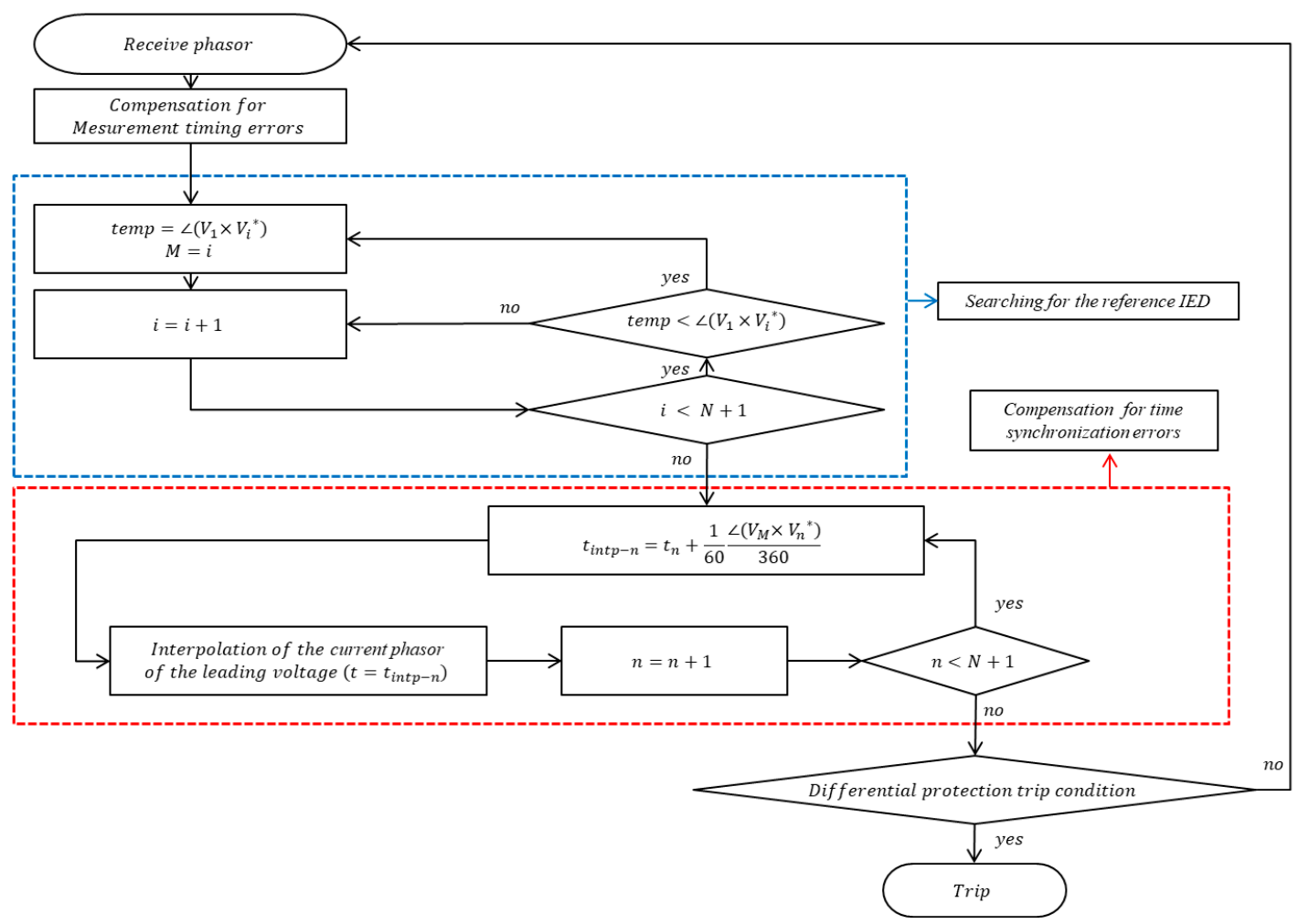

Figure 8. Flowchart of centralized busbar differential protection with data desynchronization compensation. 
As the first step in data desynchronization compensation, the first-order Lagrange interpolation polynomial is used to compensate for the measurement timing errors, assuming that the time synchronization is perfect. For the second step, the time synchronization errors are compensated for by using the voltage angle differences between local IEDs. The centralized busbar differential protection is applied when data desynchronization compensation is completed.

\section{Performance Evaluation}

\subsection{Simulation Tests}

Figure 9 shows a typical Korean $154 \mathrm{kV}$ digital substation modeled in PSCAD/EMTDC. The primary side of the $154 / 22.9 / 6.6 \mathrm{kV}$ transformer is grounded via a lightning arrester (LA); the secondary side is grounded via a neutral grounding reactor (NGR); and the tertiary side is ungrounded. A 40 MVA initial load is applied to the $22.9 \mathrm{kV}$ busbar. Table 2 lists the modeling parameters used in PSCAD/EMTDC, which are defined in the RTDS-based procedure for testing the IEDs to be used in KEPCO $154 \mathrm{kV}$ substations [39].

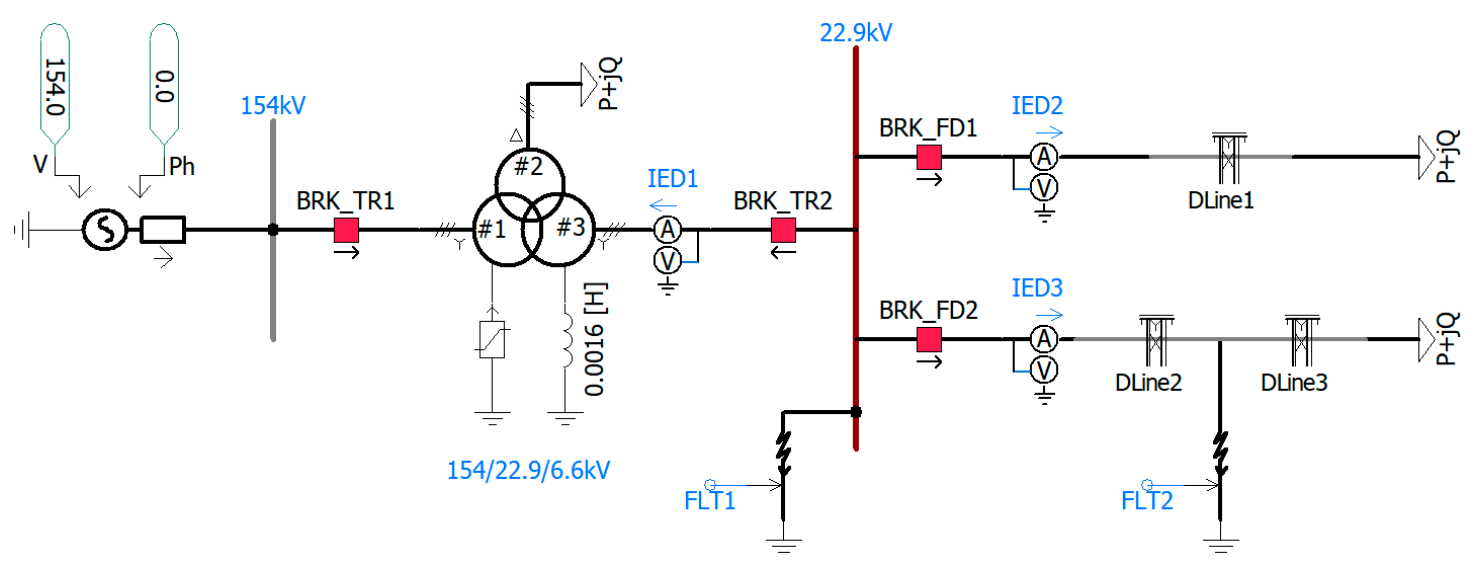

Figure 9. A typical, Korean $154 \mathrm{kV}$ digital substation modeled in PSCAD/EMTDC.

Table 2. Modeling parameters of a $154 / 22.9 / 6.6 \mathrm{kV}$ digital substation.

\begin{tabular}{|c|c|c|}
\hline Type & \multicolumn{2}{|c|}{ Parameter (\%Z, 100 MVA Base) } \\
\hline Source $(154 \mathrm{kV})$ & $R 1+j X 1, R 0+j X 0$ & $0.081+j 0.866,0.384+j 1.956$ \\
\hline \multirow[t]{2}{*}{$\begin{array}{l}\text { Transformer } \\
\text { (Y-Y-D) }\end{array}$} & 154/22.9/6.6 kV (3-phase 60 MVA) & $\begin{array}{l}\% Z 12=j 31 \% \\
\% Z 13=j 50 \% \\
\% Z 23=j 13 \%\end{array}$ \\
\hline & LA, NGR grounding & $\operatorname{NGR}(j 34 \%)=0.6 \Omega$ \\
\hline Feeder(22.9 kV) & $R 1+j X 1, R 0+j X 0$ & $0.1+j 34,0+j 47$ \\
\hline Load & $20 \mathrm{MVA} * 2, \mathrm{P} . \mathrm{F}=0.9$ & $\begin{array}{c}\mathrm{R}=47.61 \Omega \\
\mathrm{L}=0.0611 \mathrm{H}\end{array}$ \\
\hline
\end{tabular}

Figure 10 shows the effect of data desynchronization on the differential current when an external fault develops in FLT2 of Figure 9. The simulation result for a data synchronization error of $0 \mathrm{~ms}$ corresponds to conventional differential protection; the result is the same as that of the centralized differential protection proposed in this paper. Please note that no additional investment is required if the proposed protection is implemented; in contrast, conventional differential protection requires an IED and several current transformers. If the data synchronization error is $0.5 \mathrm{~ms}, 10 \%$ of the external fault current is calculated as the differential current; if the data synchronization errors are 1.0 and $5.0 \mathrm{~ms}$, the differential currents are approximately 30 and $150 \%$ of the external fault currents, respectively. As the minimum pickup offset is set to $10 \%$ of the external fault current, the centralized differential 
protection without data desynchronization compensation will be erroneously operated when the data synchronization error is larger than $0.5 \mathrm{~ms}$. Therefore, data desynchronization compensation is essential to prevent erroneous operation of centralized differential protection.

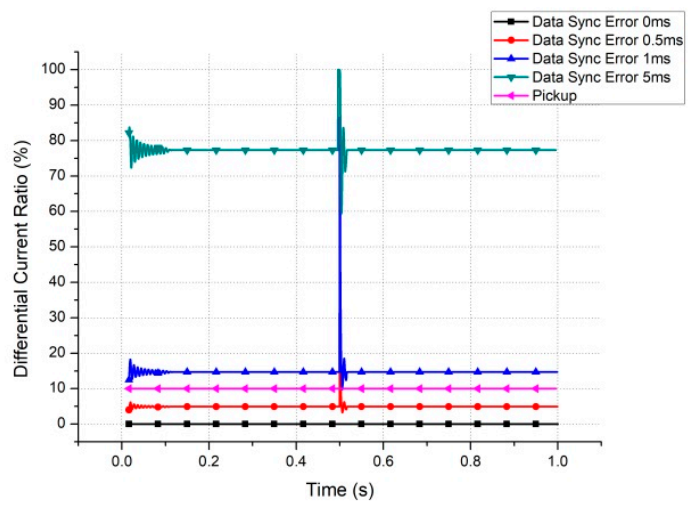

(a)

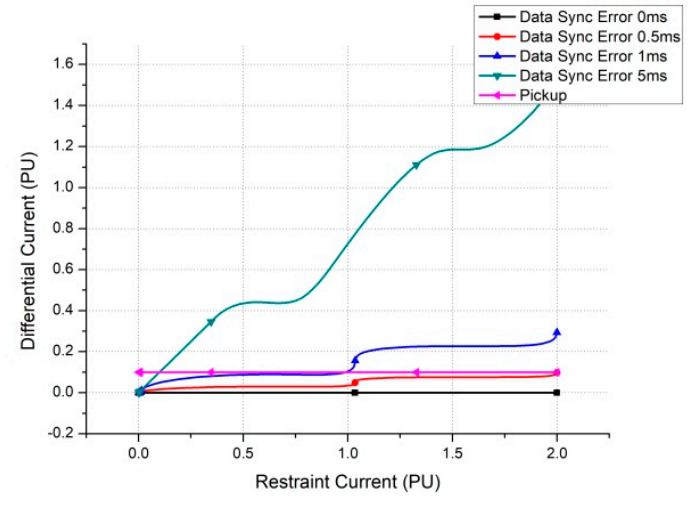

(b)

Figure 10. Differential current errors caused by data desynchronization in the case of an external fault: (a) The differential current ratio and, (b) the operating curve of a busbar, differential protection scheme.

\subsection{Hardware Tests}

\subsubsection{Test Environment}

IEC 61850-based local IEDs were implemented using the Smart Grid Infrastructure EValuation Module (SGIEVM), which is a development platform for smart grid infrastructure applications that includes a data concentrator, protection scheme, and substation automation. The SGIEVM is based on an OMAP-L138 processor, which is a dual-core system-on-a-chip (SoC) with one Acorn RISC Machine (ARM) core and a digital signal processor (DSP). To consider time synchronization errors, an SNTP time server was implemented, which transmitted a time signal to local IEDs. Each local IED synchronized its time reference according to the time signal and transmitted its phasor data with a timestamp to a central IED every $50 \mathrm{~ms}$ using SV. As mentioned in Section 2.2, five ASDUs were contained in each SV frame. As shown in Figure 11, the test environment includes a Real-Time Digital Simulator (RTDS), three local IEDs, and a central IED. Although the analog-to-digital converter (ADC) on the SGIEVM accepted inputs between 0 and $240 \mathrm{~V}$, the RTDS can produce voltage signals only below $10 \mathrm{~V}$ corresponding to the fault currents. Therefore, some additional errors could be caused by the limited resolution of the ADC across the input range used.

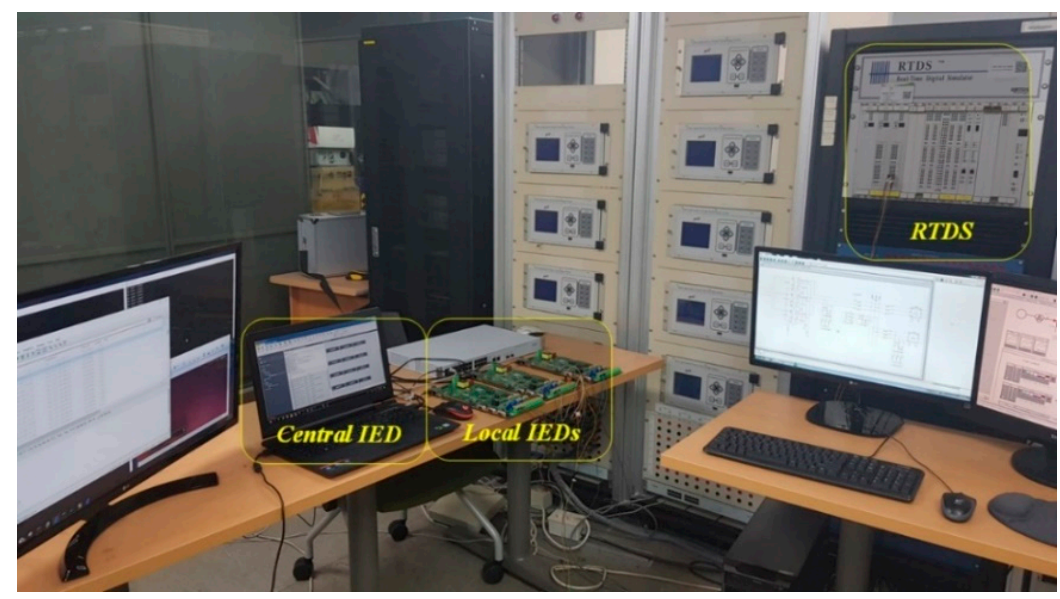

Figure 11. Test environment for the centralized busbar differential protection. 
Figure 12 shows the Korean $154 \mathrm{kV}$ digital substation modeled in the RTDS, including a schematic of data exchange. The RTDS used the same system configuration and modeling parameters as the PSCAD/EMTDC. The testing in this paper was proceeded according to the RTDS-based procedure for testing the IEDs to be used in KEPCO $154 \mathrm{kV}$ substations [39].

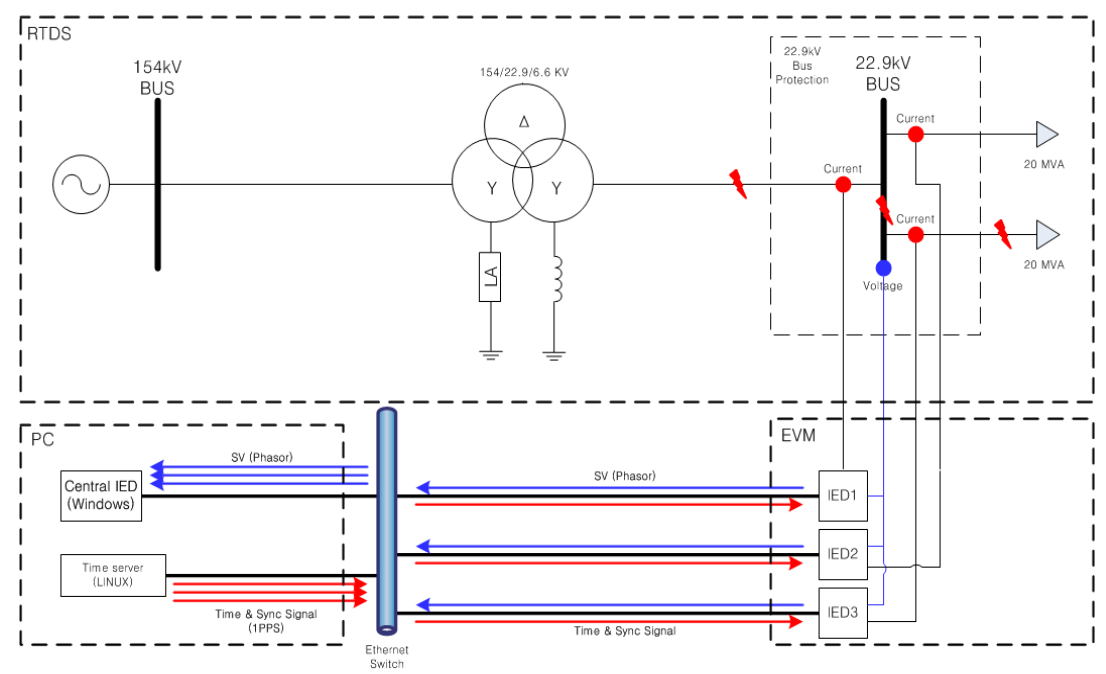

Figure 12. Schematic for testing the centralized busbar differential protection.

\subsubsection{Case Studies}

The $22.9 \mathrm{kV}$ centralized busbar differential protection was tested under normal and fluctuating load conditions. Figure 13 shows the differential current ratios under normal load conditions. Since there was no fault, the differential current ratio should be zero; however, when the centralized busbar differential protection was applied without data desynchronization compensation, considerable differential current ratios were calculated over the pickup ratio, leading to erroneous operation. Fortunately, the data desynchronization compensation reduced the differential current errors to considerably below the pickup ratio and enabled successful operation of the centralized busbar differential protection.

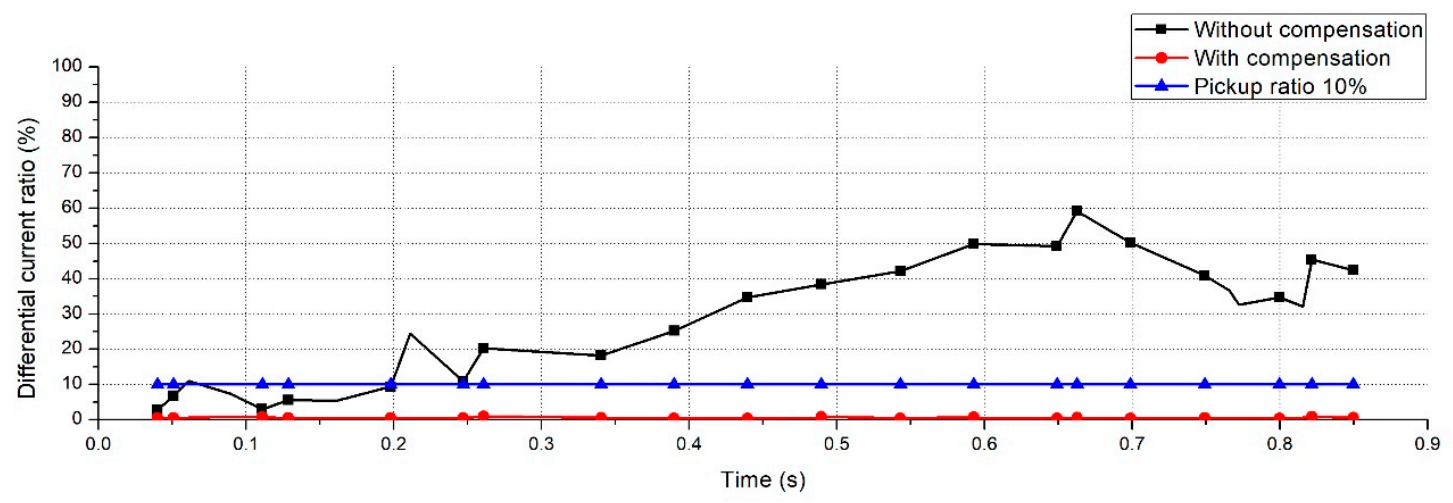

Figure 13. Test case under normal load conditions.

Figure 14 shows the differential current ratios under fluctuating load conditions. The total load was increased by $20 \%$ for $1 \mathrm{~s}$, and then decreased by $20 \%$ for the next $1 \mathrm{~s}$. Similar to the normal load conditions, the data desynchronization compensation reduced the differential current errors to considerably below the pickup ratio. 


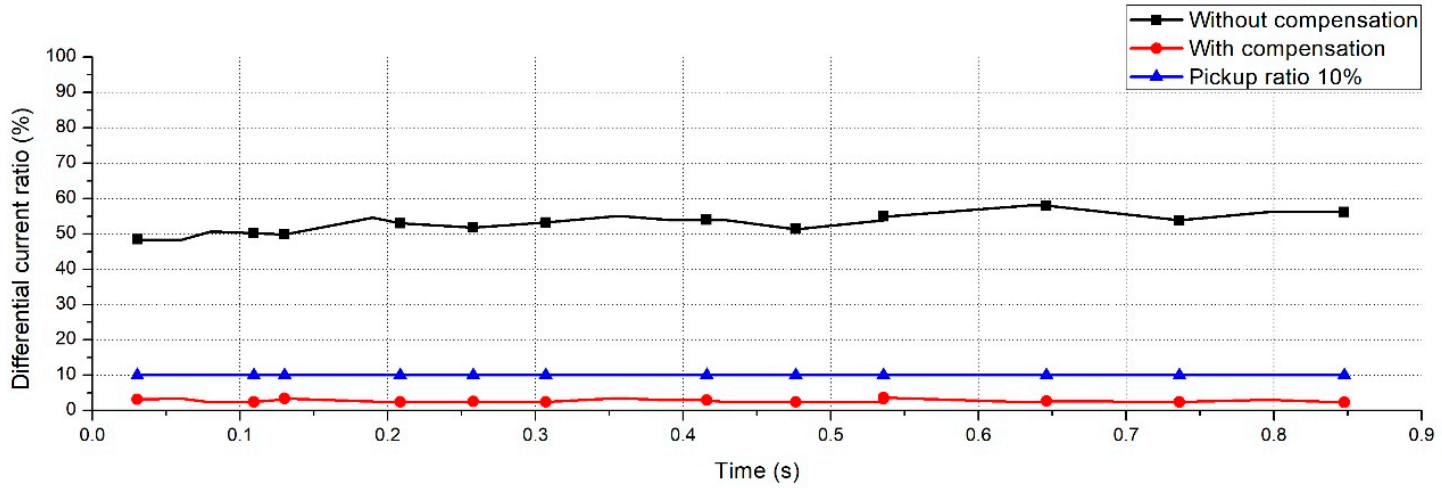

Figure 14. Test case under fluctuating load conditions.

Figure 15 shows the results of an external fault that occurred on the feeder side. When the external fault occurred, the centralized busbar differential protection experienced a transient state with a duration of about $0.08 \mathrm{~s}$. During the transient state, differential current errors occurred due to the interpolation errors between local IEDs. Although the differential current ratio was increased over the pickup ratio during the transient state, it was decreased to below the pickup ratio when the post-steady state was reached.

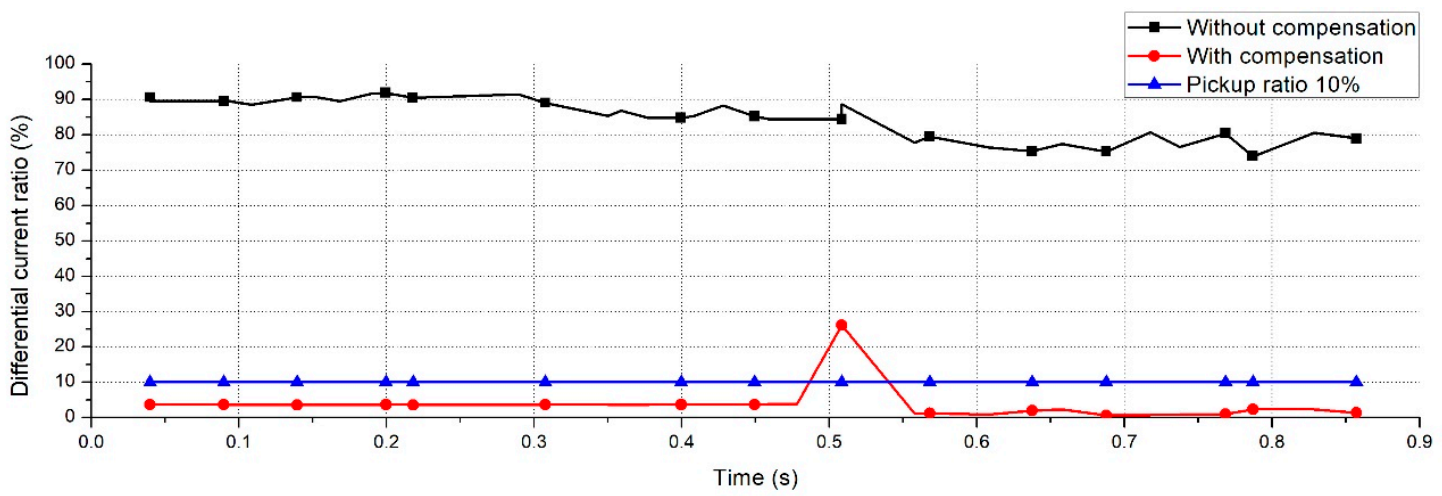

Figure 15. Test case for a line-to-ground external fault on the feeder side under normal load conditions.

Figure 16 shows the results of an external fault that occurred on the secondary side of the transformer. Similar to the external fault on the feeder side, the external fault caused a transient state with a duration of about $0.08 \mathrm{~s}$. Although the differential current ratio was increased over the pickup ratio during the transient state, it was decreased to below the pickup ratio when the post-steady state was reached.

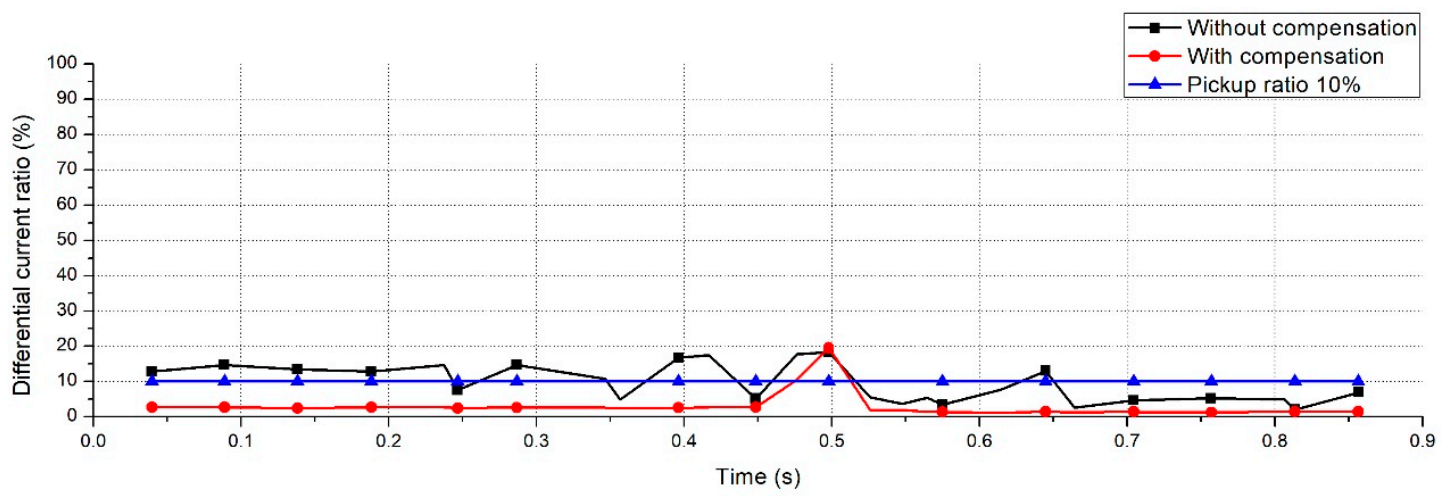

Figure 16. Test case for a line-to-ground external fault on the secondary side of the transformer under normal load conditions. 
Figure 17 shows the results of an internal fault on the $22.9 \mathrm{kV}$ busbar. As the differential current ratio converged to a stable value over the pickup ratio, the centralized busbar differential protection was operated, and the internal fault was detected successfully.

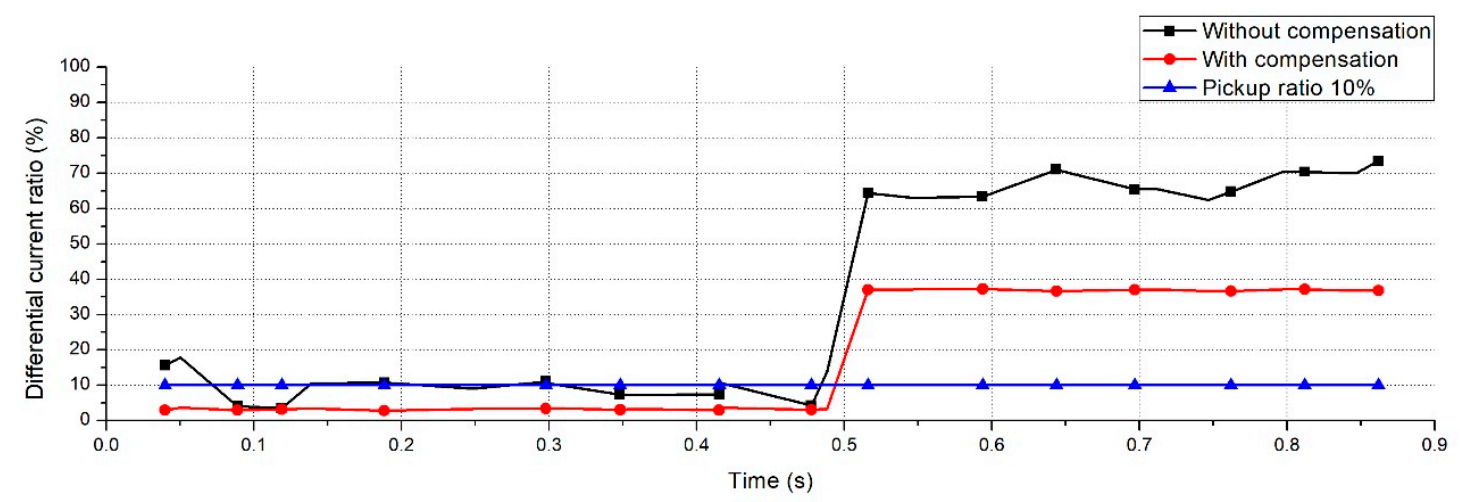

Figure 17. Test case for a line-to-ground internal fault on the $22.9 \mathrm{kV}$ busbar under normal load conditions.

Figure 18 shows the compensation errors in the case of the internal fault. After the transient state, the centralized busbar differential protection scheme detects the internal fault successfully. Therefore, the centralized busbar differential protection scheme can be used as backup protection in a centralized environment.

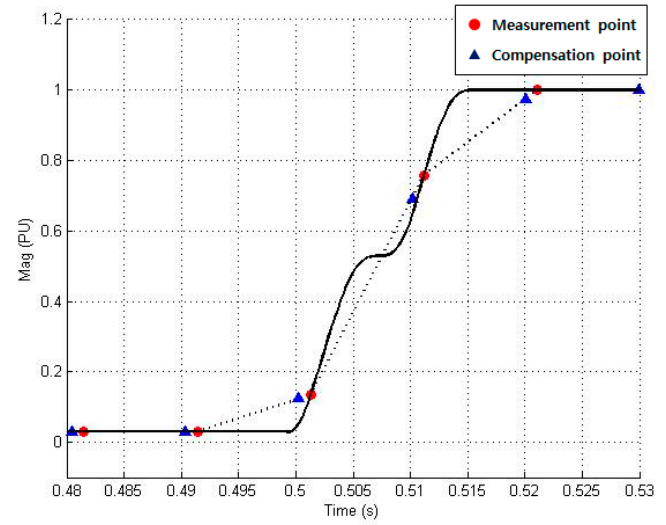

(a)

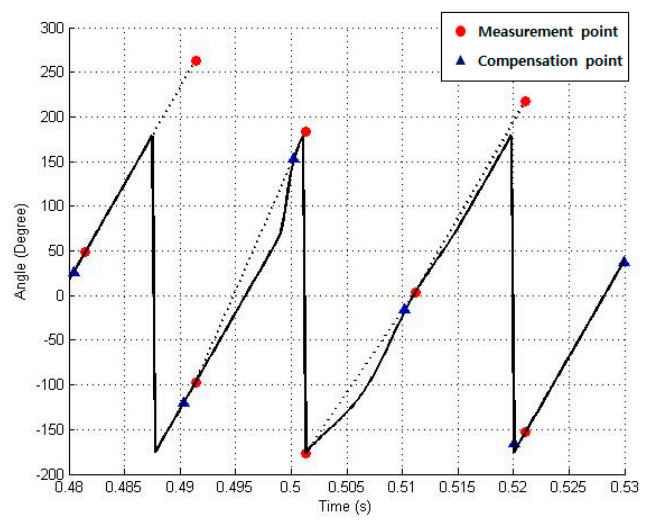

(b)

Figure 18. Analysis of the compensation errors due to an internal line-to-ground fault: (a) magnitude and (b) phase angle compensation errors

\section{Conclusions}

This paper has proposed a centralized busbar differential protection scheme as backup protection for centralized environments, such as a digital substation where data desynchronization occurs among IEC 61850-based IEDs. The centralized backup protection can provide additional protection for $22.9 \mathrm{kV}$ busbars that at present are simply protected by over-current protection in South Korea. In this paper, measurement timing and time synchronization errors were considered as the main causes of data desynchronization. The first-order Lagrange interpolation polynomial was used to compensate for the measurement timing errors, and the voltage angle differences between local IEDs were used to compensate for the time synchronization errors. As exchange of instantaneously sampled data could lead to severe network traffic, each local IED transmitted its phasor data to the central IED every 50 ms using SV. Five ASDUs were contained in each SV frame to improve the accuracy of interpolation 
used to compensate for the measurement timing errors. The central IED compensated for the data desynchronization and then performed the centralized busbar differential protection.

The centralized busbar differential protection was tested using a real-time digital simulator and IEC 61850-based local IEDs. To consider the desynchronization environment, SNTP was used for time synchronization, and no external trigger signal was used for measurement timing synchronization. The test results showed that the data desynchronization compensation can significantly reduce differential current errors and consequently prevent erroneous operation of the IEC 61850-based centralized busbar differential protection scheme. In the future, it is expected that the methodology used in this paper would be expanded to cover the centralized backup protection in digital substations.

Author Contributions: Conceptualization, M.-H.S. and S.-R.N.; Methodology, M.-H.S., S.-H.K., N.-H.L. and S.-R.N.; Supervision, S.-R.N.; Validation, M.-H.S.; Writing-original draft, M.-H.S.; Writing—review \& editing, S.-H.K., N.-H.L. and S.-R.N. All authors have read and agreed to the published version of the manuscript.

Funding: This research was supported in part by the Human Resources Program in Energy Technology of the Korea Institute of Energy Technology Evaluation and Planning (KETEP), and granted financial resources from the Ministry of Trade, Industry \& Energy, Republic of Korea (No. 20154030200770). This research was also supported in part by Korea Electric Power Corporation (Grant number: R17XA05-2).

Conflicts of Interest: The authors declare no conflict of interest.

\section{References}

1. International Electrotechnical Comission (IEC). Communication Networks and System for Utility Automation-Part 1: Introduction and Overview, 2nd ed.; IEC 61850-1; IEC: Geneva, Switzerland, 2012.

2. Silos, A.; Senis, A.; De Pozuelo, R.M.; Zaballos, A. Using IEC 61850 GOOSE Service for Adaptive ANSI 67/67N Protection in Ring Main Systems with Distributed Energy Resources. Energies 2017, 10, 1685. [CrossRef]

3. Jurisic, G.; Havelka, J.; Capuder, T.; Sucic, S. Laboratory Test Bed for Analyzing Fault-Detection Reaction Times of Protection Relays in Different Substation Topologies. Energies 2018, 11, 2482. [CrossRef]

4. Ustun, T.S.; Ozansoy, C.; Zayegh, A. Modeling of a Centralized Microgrid Protection System and Distributed Energy Resources According to IEC 61850-7-420. IEEE Trans. Power Syst. 2012, 27, 1560-1567. [CrossRef]

5. Monadi, M.; Gavriluta, C.; Luna, A.; Candela, J.I.; Rodriguez, P. Centralized Protection Strategy for Medium Voltage DC Microgrids. IEEE Trans. Power Deliv. 2017, 32, 430-440. [CrossRef]

6. Deng, W.; Pei, W.; Shen, Z.; Zhao, Z.; Qu, H. Adaptive Micro-Grid Operation Based on IEC 61850. Energies 2015, 8, 4455-4475. [CrossRef]

7. Wang, Y.; Syed, M.H.; Guillo-Sansano, E.; Xu, Y.; Burt, G.M. Inverter-Based Voltage Control of Distribution Networks: A Three-Level Coordinated Method and Power Hardware-in-the-Loop Validation. IEEE Trans. Sustain. Energy 2019. [CrossRef]

8. Kim, S.K. The Situation and Future of the Technology for IEC 61850 Based Substation Automation System in Korea. In Proceedings of the 18th IERE General Meeting and Japan Forum, Kyoto, Japan, 21-24 May 2018.

9. Yi, Y.H.; Zhang, J.T.; Liu, B.; Xu, L.Z.; Cao, Y.J.; Guo, C.X. A new-style centralized IED based on IEC 61850. In Proceedings of the IEEE Power and Energy Society General Meeting-Conversion and Delivery of Electrical Energy in the 21st Century, Pittsburgh, PA, USA, 20-24 July 2008.

10. Li, Q.; Zhou, Z.; Du, D.; Li, Z.; Li, W.; Wang, X. A novel substation area backup protection for smart substation. In Proceedings of the 2013 IEEE PES Asia-Pacific Power and Energy Engineering Conference (APPEEC), Kowloon, China, 8-11 December 2013.

11. Ali, I.; Hussain, S.M.S.; Tak, A.; Ustun, T.S. Communication Modeling for Differential Protection in IEC-61850-Based Substations. IEEE Trans. Ind. Appl. 2018, 54, 135-142. [CrossRef]

12. Gao, H.; Liu, Y.; Zou, G.; Cui, D.; Liu, M.; Li, X. Principle and implementation of substation-area backup protection for digital substation. In Proceedings of the 12th IET International Conference on Developments in Power System Protection (DPSP 2014), Copenhagen, Denmark, 31 March-3 April 2014.

13. Li, Y.; Gao, H.; Gao, W.; Peng, F. Development of a Substation-Area Backup Protective Relay for Smart Substation. IEEE Trans. Smart Grid 2017, 8, 2544-2553. [CrossRef]

14. Brahma, S. Advancements in Centralized Protection and Control Within a Substation. IEEE Trans. Power Deliv. 2016, 31, 1945-1952. [CrossRef] 
15. Albinali, H.F.; Meliopoulos, A.P.S. Resilient Protection System Through Centralized Substation Protection. IEEE Trans. Power Deliv. 2018, 33, 1418-1427. [CrossRef]

16. Hohn, F.; Rabuzin, T.; Wang, J.; Nordstrom, L. Distributed signal processing units for centralised substation protection and control. J. Eng. 2018, 2018, 1223-1228. [CrossRef]

17. Li, W.; Tan, Y.; Li, Y.; Cao, Y.; Chen, C.; Zhang, M. A New Differential Backup Protection Strategy for Smart Distribution Networks: A Fast and Reliable Approach. IEEE Access 2019, 7, 38135-38145. [CrossRef]

18. Teoh, C.P.; Newman, P.; Lloyd, G.; Qin, H.; Hunt, R.; Mendez, J.; Smith, T. A Solution to Eliminate Conventional Busbar Protection with Process Bus. In Proceedings of the Relay Conference, College Station, TX, USA, 25-28 March 2019.

19. Orr, P.; Gordon, N.; Clayburn, L.; Ma, Z.; Hong, Q.; Tzelepis, D.; Hurzuk, N.S.; Loken, R.; Booth, C. Implementation of centralised numerical busbar protection using distributed photonic current sensors. In Proceedings of the PAC World Conference, Glasgow UK, 17-20 June 2019.

20. IEC/IEEE Standard for Precision Time Protocol Profile for Power Utility Automation; IEC/IEEE 61850-9-3; IEC: Geneva, Switzerland, 2016.

21. IEC Technical Report for Use of Logical Nodes for Modeling Application Functions and Related Concepts and Guidelines for Substations, 1st ed.; IEC TC-57, IEC TR 61850-7-500; IEC: Geneva, Switzerland, 2017.

22. Jv, H.; Gao, X.H. Application of Time Synchronization System Based on SNTP in Digital Substation. In Proceedings of the 2008 China International Conference on Electricity Distribution (CICED), Guangzhou, China, 10-13 December 2008.

23. Ussoli, M.; Prytz, G. SNTP time synchronization accuracy measurements. In Proceedings of the 2013 IEEE 18th Conference on Emerging Technologies \& Factory Automation (ETFA), Guangzhou, China, 10-13 December 2013.

24. Manembu, P.; Kewo, A.; Welang, B. Missing data solution of electricity consumption based on Lagrange Interpolation case study: IntelligEnSia data monitoring. In Proceedings of the 2015 International Conference on Electrical Engineering and Informatics (ICEEI), Denpasar, Indonesia, 10-11 August 2015.

25. Song, M.H.; Lee, N.H.; Nam, S.R. A study on IEC 61850 based Centralized 22.9kV Bus Protection considering Time Synchronization Errors. Trans. Korean Inst. Electr. Eng. 2019, 68, 965-971. [CrossRef]

26. Cai, Y.; Chen, Y.; Li, Y.; Cao, Y.; Zeng, X. Reliability Analysis of Cyber-Physical Systems: Case of the Substation Based on the IEC 61850 Standard in China. Energies 2018, 11, 2589. [CrossRef]

27. Wannous, K.; Toman, P.; Jurak, V.; Wasserbauer, V. Analysis of IEC 61850-9-2LE Measured Values Using a Neural Network. Energies 2019, 12, 1618. [CrossRef]

28. Leon, H.; Montez, C.; Valle, O.; Vasques, F. Real-Time Analysis of Time-Critical Messages in IEC 61850 Electrical Substation Communication Systems. Energies 2019, 12, 2272. [CrossRef]

29. Kanabar, M.G.; Sidhu, T.S. Performance of IEC 61850-9-2 Process Bus and Corrective Measure for Digital Relaying. IEEE Trans. Power Deliv. 2011, 26, 725-735. [CrossRef]

30. Ingram, D.M.E.; Schaub, P.; Taylor, R.R.; Campbell, D.A. Performance Analysis of IEC 61850 Sampled value Process Bus Networks. IEEE Trans. Ind. Inform. 2013, 9, 1445-1454. [CrossRef]

31. SISCO. MMS Lite-IEC 61850 for Embedded Systems. Available online: https://sisconet.com/wp-content/ uploads/2019/04/MktLit_MMS-Lite_01232018.pdf (accessed on 20 February 2020).

32. TI. SGI EVM Hardware Manual. Available online: http://processors.wiki.ti.com/index.php/SGI_EVM_ Hardware_Manual (accessed on 20 February 2020).

33. IEC Standard for Specific Communication Service Mapping (SCSM)—Mappings to MMS (ISO/IEC 9506-1 and ISO/IEC 9506-2) and to ISO/IEC 8802-3, 2nd ed.; IEC TC-57, IEC 61850-8-1; IEC: Geneva, Switzerland, 2011.

34. IEC Standard for Communication Networks and Systems for Power Utility Automation -Part 7-1: Basic Communication Structure-Principles and Models, 2nd ed.; IEC TC-57, IEC 61850-7-1; IEC: Geneva, Switzerland, 2011.

35. IEC Standard for Communication Networks and Systems for Power Utility Automation-Part 9-2: Specific Communication Service Mapping (SCSM)—Sampled Values over ISO/IEC 8802-3, 2nd ed.; IEC TC-57, IEC 61850-9-2; IEC: Geneva, Switzerland, 2009.

36. IEC Standard for Communication Networks and Systems for Power Utility Automation-Part 7-3: Basic Communication Structure-Common Data Classes, 2nd ed.; IEC TC-57, IEC 61850-7-3; IEC: Geneva, Switzerland, 2009.

37. IEC Standard for Communication Networks and Systems for Power Utility Automation-Part 7-4: Basic Communication Structure-Compatible Logical Node Classes and Data Object Classes, 2nd ed.; IEC TC-57, IEC 61850-7-4; IEC: Geneva, Switzerland, 2009. 
38. Christoph, S.B.; Gerhard, L.; Frederic, L.; Fred, F. Implementation Guideline for Digital Interface to Instrument Transformers Using IEC 61850-9-2; UCA International Users Group: Raleigh, NC, USA, 2004.

39. Power System Protection Team. RTDS-Based Procedure for Testing the IEDs to be Used in KEPCO 154-kV Substations; KEPCO: Naju, Korea, 2012.

(C) 2020 by the authors. Licensee MDPI, Basel, Switzerland. This article is an open access article distributed under the terms and conditions of the Creative Commons Attribution (CC BY) license (http://creativecommons.org/licenses/by/4.0/). 